\title{
A Bi-functional Polymer with Electro-chromic and Self-healing Performance
}

\author{
Rongzong Zheng ${ }^{\text {a }}$, Yaru Fan ${ }^{b}$, Yi Wang ${ }^{\text {, }}$, Chunyang Jia*, Jianliang Xie ${ }^{d}$ \\ State Key Laboratory of Electronic Thin Films and Integrated Devices, National Engineering Research \\ Center of Electromagnetic Radiation Control Materials, School of Electronics Science and Engineering, \\ University of Electronic Science and Technology of China, Chengdu 610054, China \\ *cyjia@uestc.edu.cn, ${ }^{\text {a }}$

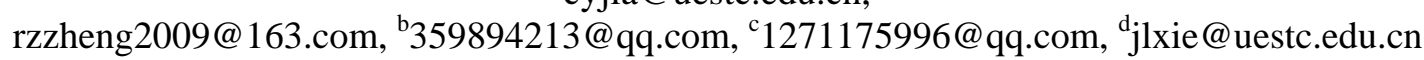

Keywords: Self-healing Performance, Electro-chromic, Bi-functional Polymer

\begin{abstract}
The cracking of electrochromic materials due to aging or reiterative bending is a major problem, which noticeably degrades the performance of electrochromic devices. In this paper, a new polymer DFTPA-MA, which integrate the triphyelamine (TPA) and self-healing group, is reported in this paper. The colour of the polymer not only could switch colours between light yellow and orange red, and also have the good self-healing properties for 5 min to heal the crack about $10 \mu \mathrm{m}$. The polymer DFTPA-MA may hold great potential to overcome the scars generation on the electrochromic devices.
\end{abstract}

\section{Introduction}

Electrochromism is the reversible color changes in the transmittance or/and reflectance by applying extermal voltages [1]. Currently, inorganic transition metal oxide [2], organic small molecular materials[3] and $\pi$-conjugated polymers[4] are some most widely used species of electrochromic materials. Organic electrochromic materials have gained special attention from the researchers and design engineers due to their structural modification [5] and tunable colors response[6]. Recently, more researches have gained special attention in flexible[7], stretchable[8] and even for wearable devices[9].

Despite the noteworthy progress, electrochromic devices (ECDs) are still facing challenges that the generation of cracks from aging or reiterative bending, which was inveitable with passage of time. No doubt that the cracks would strongly degrades the performance of the ECDs, and hence limits the overall efficiency and active life of ECDs. So repairing cracks timely would be vital for prolonging the service life of ECD. However, traditional electrochromic materials are not intrinsically healable.

Self-healing materials[10], is also a kind of smart material, which have the intrinsic capacity to repair the cracks to obviate such risks and thereby lead to reduced waste, improve lifetime, durability of the devices. It is believed that the electrochromic polymer film with self-healing properties would be an effective approach to overcome the risk of the scars generation in ECDs.

Until now, self-healing polymers based on Diels-Alder(DA) reaction, which is an effective method for the implementation of intrinsic self-healing into function materials, have been deeply studied for various electronic and optoelectronic applications like conductors films[11], OLEDs[12] and supercapacitors[13] etc. These materials can repair the cracks by retro-DA and DA reaction only by means of heat treatment without catalyst or other special treatment. It was the first time to make the electrochromic films curable by themselves when be damaged.

In this paper, a novel polymer DFTPA-MA, which has electrochromic and self-healing properties, was synthesized and reported. As describe in Figure 1, the TPA derivative was the electrochromic unit and induced into the furfuryl glycidyl ether (FGE), and then DA cross-link polymerize with maleimide (MA) to obtain the nonconjugated TPA-based polymer DFTPA-MA. It not only exhibits electrochromic properties but also possess self-healing nature, which indicate DFTPA-MA is a promising material to overcome the cracking problems in ECDs. 


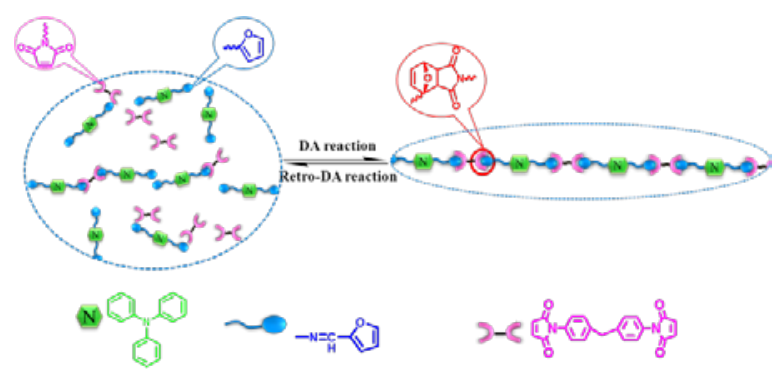

Fig 1. The synthetic route of polymer DFTPA-MA.

\section{Experiment}

\subsection{Materials and reagents}

The ITO (15 $\Omega / \mathrm{cm}^{2}$, commercially purchased) coated glass substrate was cut into pieces with size of $0.8 \times 4 \mathrm{~cm}^{2}$, then ultrasonic washed with acetone, ethanol, deionized water for 15 minutes, respectively, and then immerged in ethanol for use. $0.1 \mathrm{~mol} \cdot \mathrm{L}^{-1}$ TBAP in acetonitrile $\left(\mathrm{CH}_{3} \mathrm{CN}\right)$ was employed as electrolyte in the test. Other reagents and solvents were purchased from commercial sources and used without any further purification.

\subsection{Instruments}

The infrared spectrum was observed by the FT-IR (8400s, SHI-MADZU), high resolution mass spectra (HRMS) data was determined with an FTICR-APEX instrument, nuclear magnetic resonance (NMR) spectra was measured on Brücker AM 400 NMR instrument.

The electrochemical analysis of polymer DATPFMA film, which coated on the ITO glass substrate, was measured by cyclic voltammery (CV) on CHI660C electrochemical workstation in electrolyte solution, the reference electrode was $\mathrm{Ag} / \mathrm{AgCl}$ in saturated and $\mathrm{Ti}$ sheet as the counter electrode.Optical property and kinetic feature of polymer DATPFMA film was observed on spectrophotometer (UV-2250, SHI-MADZU) and CHI660 electrochemical workstation. A situ colorimeter (SP60, X-Rite) was used to test chromaticity of the polymer film, which was switched to different states by amperometric i-t method on the electrochemical workstation before tests, each states of the film was tested at least three times to approximate average values. Differential scanning calorimetry (DSC) analyses were carried out on Q200 (TA, USA) at a heating rate of $10^{\circ} \mathrm{Cmin}^{-1}$ in flowing of nitrogen (flow rate $50 \mathrm{~cm}^{3} \mathrm{~min}^{-1}$ ), and the enthalpy value $(\Delta \mathrm{H})$ was investigated by the Universal Analysis 2000.

\subsection{Synthesis}

The synthetic route of polymer DFTPA-MA is shown in Scheme 1, and the N-(4-aminophenyl)N-phenylbenzene-1,4-diamine (DATPA) was synthesized according to literature[14].

Synthesis of the monomer DFTPA: DATPA $(0.28 \mathrm{~g})$ and furan-2-carbaldehyde $(0.22 \mathrm{~g})$ were added into a flask and stirred under nitrogen protection, then the mixture was kept $95{ }^{\circ} \mathrm{C}$ for $24 \mathrm{~h}$, then residual furan-2-carbaldehyde was evaporated to give a brownish solid, and purified by a column chromatography to obtain yellowish powder $(\eta=83 \%) .{ }^{1} \mathrm{H}$ NMR (400 MHz, D6-DMSO) $\delta$ : 7.51(s, 2H), 7.35(d, 2H), 6.94-7.01(m, 6H), 6.70-6.74(d, 2H), 6.47-6.56(m, 3H), 6.43-6.45(d, 2H), 6.28-6.31(m, 4H). MS-ESI (m/z): calcd for $\left(\mathrm{C}_{28} \mathrm{H}_{21} \mathrm{~N}_{3} \mathrm{O}_{2}\right)$ : 431.1634; found $431.8257[\mathrm{M}+\mathrm{H}]^{+}$. FTIR (KBr) v $\left(\mathrm{cm}^{-1}\right)$ : 2931, 2339, 1659, 1594, 1494, 1392, 1268, 1154, 1074, 747, 688, 594.

Preparation of the polymer DFTPA-MA film: DFTPA (0.43 g) and MA (0.36 g) were dissolved into $2 \mathrm{~mL}$ cyclopentanone, then the mixture was stirred at $110^{\circ} \mathrm{C}$ for $4 \mathrm{~h}$ to obtain the luminous yellow viscous liquid, then coated onto the clean ITO glass substrate and dried at $80^{\circ} \mathrm{C}$ for $6 \mathrm{~h}$ to get the homogeneous polymer DFTPA-MA film $(\sim 5 \mu \mathrm{m})$. 


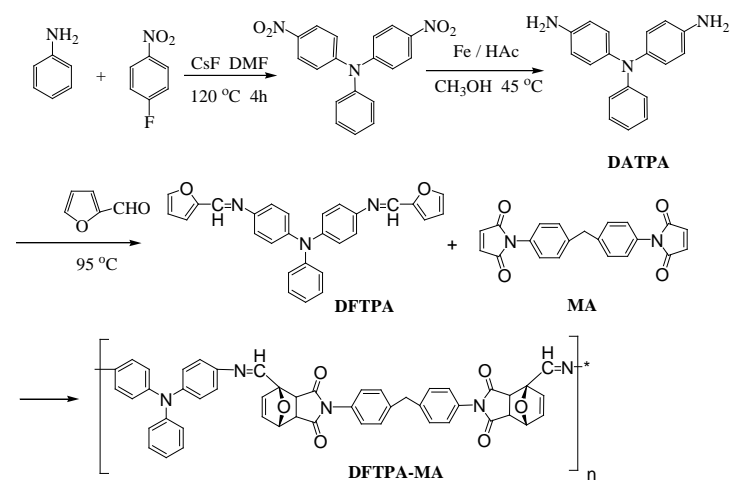

Fig. 2 The synthetic route of polymer DFTPA-MA.

\section{Results and Discussion}

\subsection{Electrochromic properties}

The electrochemical behaviour of the polymer DFTPA-MA film was investigated by cyclic voltammogram $(\mathrm{CV})$ ranges from 0 to $1.0 \mathrm{~V}$, the $\mathrm{CV}$ curve as shown in the Figure 2a. A couple redox peaks were observed from the $\mathrm{CV}$ circle, the redox peaks at $\mathrm{E}_{\mathrm{ox}}=0.66 \mathrm{~V}, \mathrm{E}_{\mathrm{red}}=0.53 \mathrm{~V}$, respectively.

The colour of the polymer DFTPA-MA film was changed from light yellow to orange red as the applied voltage values of $0 \mathrm{~V}$ and $0.7 \mathrm{~V}$, respectively. Colors of the polymer film were measured by colorimetric analysis, three parameters $\mathrm{L}^{*}$ (luminance), $\mathrm{a}^{*}$ (hue), $\mathrm{b}^{*}$ (saturation) are used to quantitatively define. Table I presents the test parameters of the polymer DFTPA-MA film.
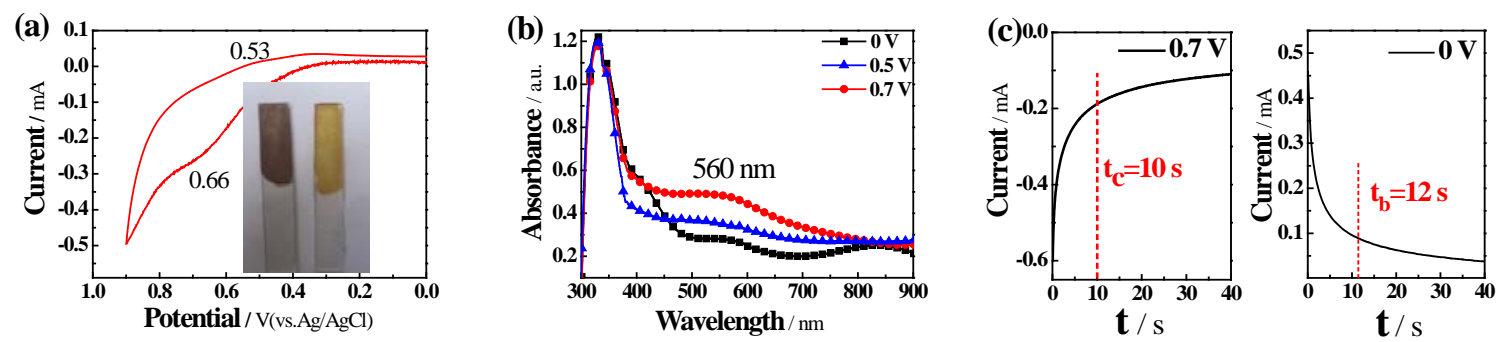

Figure 2. (a) The CV curve of polymer DFTPA-MA film at $100 \mathrm{mV} / \mathrm{s}$. (b) UV-Vis absorption spectra of polymer DFTPA-MA at different voltages, (c) the current-time curve at different voltages.

As shown in the Figure 2b, the absorbance spectra curves were recorded at different applied voltages. The sharp peak about $340 \mathrm{~nm}$ was the characteristic absorption for the $\pi-\pi^{*}$ transition of the aromatic phenyl ring of the TPA group. Once the polymer DFTPA-MA film was driven to oxidized states by applying higher voltages, a board absorption peak located at about $560 \mathrm{~nm}$ on visible region was significant changed, that attribute tocharge transfer of the central nitrogen atom of TPA group during the oxidation[6].

Table 1. Color parameters and pictures of DFTPA-MA film.

\begin{tabular}{lccccc}
\hline voltage & $\mathbf{L}^{*}$ & $\mathbf{a}^{*}$ & $\mathbf{b}^{*}$ & color & Picture \\
\hline $0 \mathrm{~V}$ & 70.91 & 4.65 & 60.36 & Light yellow & \\
$0.7 \mathrm{~V}$ & 50.03 & 7.81 & 23.88 & Orange red & \\
\hline
\end{tabular}

Response time is one of the important parameters for the electrochromic materials. As shown in the Figure 2c, response time was observed by the current-time curves (90\%), which drive the polymer DFTPA-MA film to switch colors. For the coloration process, the polymer film was changed to orange red under $0.7 \mathrm{~V}$, the current is stable after $10 \mathrm{~s}$; While for the bleached process, the polymer film from orange red turned to light yellow under $0 \mathrm{~V}$, the current is stable after $12 \mathrm{~s}$. Namely, the coloration and bleaching response times were $10 \mathrm{~s}, 12 \mathrm{~s}$. 


\subsection{Self-healing performance}

To depicte the thermally reversible of the polymer DFTPA-MA, three respected cycles between $40 \sim 180{ }^{\circ} \mathrm{C}$ was measured, as shown in the Figure 3a. The cycles showed the obvious endothermic and gently exothermic transitions in the range of $100 \sim 160^{\circ} \mathrm{C}$ can be observed to the endothermic and exothermic transition of polymer DFTPA-MA, attributed to the retro-DA and DA reactions, respectively. The above results clearly demonstrate DFTPA-MA can be used as a thermal reversible and recyclable material.

Then the DSC heating traces of the polymer DFTPA-MA was tested as shown in the Figure 3b. The samples were measure by the DSC traces, a broad endothermic peaks ranging from 100 to $170^{\circ} \mathrm{C}$ were observed for the retro DA reaction to free the DFTPA and MA group. The enthalpy values $(\Delta \mathrm{H})$ of the endothermic peak were integrated and listed in the Figure 3c. The DA bond healing rate $(\eta)$ can be calculated, the calculated formula [15] was shown as the Equation (1).

$$
\eta_{\mathrm{DSC}}=\frac{\Delta \mathrm{H}_{\text {healed }}}{\Delta \mathrm{H}_{\text {pristine }}} \times 100 \%
$$

Compared the origin polymer DFTPA-MA (DA0), the self-healing rate of the DA1 and the DA2 were calculated as $80 \%, 63 \%$, respectively.
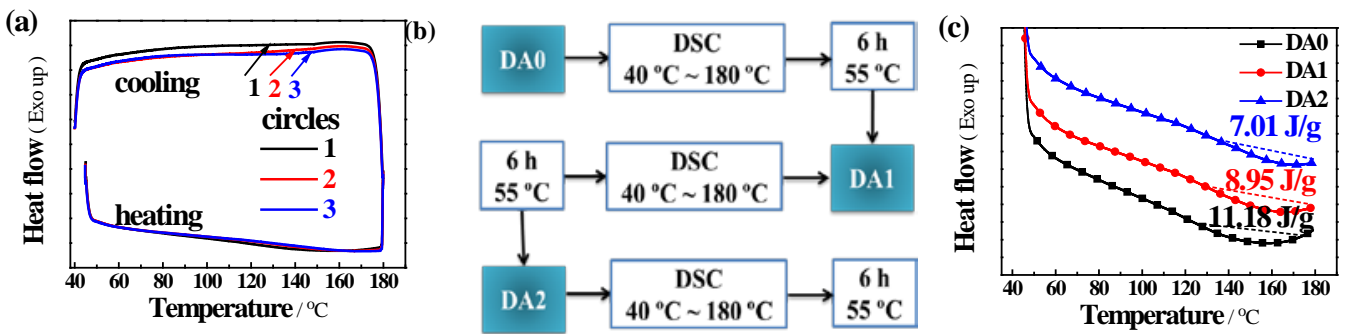

Figure 3. (a) Repeated DSC curves of polymer DFTPA-MA, (b) the procedure of testing thermal property, (c) DSC heating traces of self-healing samples DA0-2.

For detail analysis of the healing behavior, healing of the scratches on the film surface was evaluated. The polymer film (without change color) was scratched with a razor blad (about $10 \mu \mathrm{m}$ wide cuts), and the healing process at $110^{\circ} \mathrm{C}$ was observed by optical microscopy. The Figure $4 \mathrm{a}$ recorded the process of the self-healing, the scar of the polymer film started to heal at first $27 \mathrm{~s}$, the scar was blurred. $151 \mathrm{~s}$ later, the scar was essential healed and except some localized area due to the scar was too large and need more time to heal. Then the scar was completely healed after $206 \mathrm{~s}$.

(a)

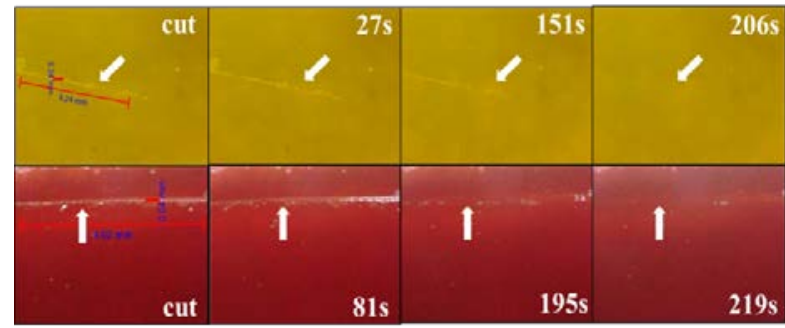

(b)

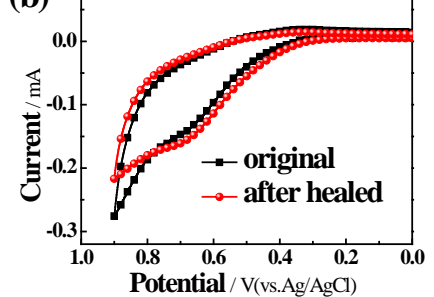

Figure 4. (a) Microscopic images of the polymer DFTPA-MA film, (b) the CV curve of polymer DFTPA-MA film before and after self-healing process.

It is clear that at applied voltage of $0.7 \mathrm{~V}$, the colors of the film vary from faint yellow to orange red. Similarly, as discussed earlier when the film was scratched and heated at $110{ }^{\circ} \mathrm{C}$, the scratched region regained its original condition. From above results, it could be seen that the self-healing process of the colored state of the polymer film was same as that of the original state, in other words, the chromatic state has no any influence for the self-healing process.

Finally, in order to analyze the effect of self-healing, the electrochromic properties of polymer DFTPA-MA films were once again investigated after self-healing at $110{ }^{\circ} \mathrm{C}$ for $5 \mathrm{~min}$. The CV curve of the healed film was tested at the same condition as the original, and the redox peaks and the area of the CV curves are similar (Figure 4b). Briefly, polymer DATPFMA has the bifunction 
without mutual influence of self-healing and electrochromism, which is due to the TPA group and the DA group is relatively independent in the molecular structure.

\section{Conclusion}

In this paper, the TPA group was induced into the furfuryl glycidyl ether and DA cross-link with MA to obtain a new bisfuction of electrochromism and self-healable polymer DFTPA-MA. The colors of the polymer film can be switched from faint yellow to green grey to dark blue, the coloring and bleaching response times were $4.1 \mathrm{~s}$ and $11.0 \mathrm{~s}$, respectively. Meanwhile, the selfhealing rate was evaluated by the enthalpy values $(\Delta \mathrm{H})$ of the DSC curves with the self-healing rate as $80 \%$. The scratch of the polymer film can be self-healed under $110^{\circ} \mathrm{C}$ during the heating process, which shows that polymer DFTPA-MA has great thermal remediable and recyclable performance. All the results indicate that the polymer DFTPA-MA film with electrochromic and self-healing properties can be as a promising material to overcome the scars generation in ECDs.

\section{Acknowledgment}

Authors are thankful to National Natural Science Foundation of China (Grant Nos. 51773027, 21572030 and 21402023) for financial support.

\section{References}

[1] R.J. Mortimer, Annu. Rev. Mater. Res. 41 (2011) 241-268.

[2] J.Z. Ou, S. Balendhran, M.R. Field, D.G. McCulloch, A.S. Zoolfakar, R.A. Rani, S. Zhuiykov, A.P. O'Mullane, K. Kalantar-Zadeh, Nanoscale 4 (2012) 5980-5988.

[3] B. Gadgil, P. Damlin, M. Heinonen, C. Kvarnström, Carbon 89 (2015) 53-62.

[4] S. L. José, R. Gómez, E. Reinold, P. Bäuerie, Org. Lett. 7 (2005) 2345-2348.

[5] P. M. Beaujuge, J. Reynolds, Chem. Rev. 110 (2010) 268-320.

[6] L. T. Huang, H. J. Yen, G. S. Liou, Macromolecules 44 (2011) 9595-9610.

[7] G. Cai, M. Cui, V. Kumar, P. Darmawan, J. Wang, X. Wang, A.L. Eh, K. Qian, P.S. Lee, Chem. Sci. 7 (2016) 1373-1382.

[8] X. Chen, H. Lin, J. Deng, Y. Zhang, X. Sun, P. Chen, X. Fang, Z. Zhang, G. Guan, H. Peng, Adv. Mater. 26 (2014) 8126-8132.

[9] C. Yan, W. Kang, J. Wang, M. Cui, X. Wang, C.Y. Foo, K.J. Chee, P.S. Lee, ACS Nano 8 (2014) 316-322.

[10] Y. Yang, M.W. Urban, Chem. Soc. Rev. 42 (2013) 7446-7467.

[11] J. Li, S. Qi, J. Liang, L. Li, Y. Xiong, W. Hu, Q. Pei, ACS Appl. Mater. Interfaces 7 (2015) 14140-14149.

[12] J. Liang, L. Li, X. Niu, Z. Yu, Q. Pei, Nature photon. 7 (2013) 817-824.

[13] Y. Huang, M. Zhong, Y. Huang, M. Zhu, Z. Pei, Z. Wang, Q. Xue, X. Xie, C. Zhi, Nature Comm. 6 (2015), 10310.

[14] X. Zhang, Y. Jin, H. Diao, F. Du, Z. Li, F. Li. Macromolecules 36 (2003) 3115-3127.

[15] P.I. Wang, W. R. Shie, J. C. Jiang, L. J. Li, D.J. Liaw, Polym. Chem. 7 (2016) 1505-1516. 